\title{
Generation of continuous-wave terahertz radiation using a two-mode titanium sapphire laser containing an intracavity Fabry-Perot etalon
}

\author{
Michael R. Stone, ${ }^{\text {a) }}$ Mira Naftaly, and Robert E. Miles \\ Institute of Microwave and Photonics, School of Electrical and Electronic Engineering, University of Leeds, \\ Leeds LS2 9JT, United Kingdom \\ Ivan C. Mayorga \\ Max-Planck Institute, Bonn D-53111, Germany \\ Andrei Malcoci \\ Institute of Microwave and Photonics, School of Electrical and Electronic Engineering, University of Leeds, \\ Leeds LS2 9JT, United Kingdom \\ Martin Mikulics \\ Institute of Thin Films and Interfaces (ISG-1), Research Center Jülich, 52425 Jülich, Germany
}

(Received 13 December 2004; accepted 21 March 2005; published online 9 May 2005)

\begin{abstract}
Continuous-wave terahertz $(\mathrm{THz})$ radiation was generated by photomixing two modes of a titanium sapphire laser. The laser was induced to oscillate on two modes by placing a Fabry-Perot etalon in the laser resonator. The frequency of terahertz radiation, which was equal to the difference frequency of the two modes, was varied by adjusting the free spectral range (FSR) of the etalon. Photomixing was performed by logarithmic spiral antennas fabricated on low-temperature-grown GaAs; and the emitted $\mathrm{THz}$ radiation was characterized. The THz power, measured by a Golay cell, was $1 \mu \mathrm{W}$ at $0.3 \mathrm{THz}$ and $0.7 \mu \mathrm{W}$ at $0.5 \mathrm{THz}$. The THz frequency, as determined by a Fourier transform interferometer, was seen to correspond to the etalon FSR. The current-voltage characteristics of photomixers were also determined, and photocurrent modulation was observed by the autocorrelation of the laser beam. (C) 2005 American Institute of Physics.
\end{abstract}

[DOI: $10.1063 / 1.1904724]$

\section{INTRODUCTION}

The generation of tuneable coherent continuous-wave $(\mathrm{CW})$ terahertz $(\mathrm{THz})$ radiation by optical heterodyne conversion in an ultrafast photoconductor was initially demonstrated by Brown et al. ${ }^{1}$ Two titanium (Ti) sapphire lasers were photomixed in a biased interdigitated electrode structure fabricated on low-temperature-grown (LT)-GaAs. This paved the way for the development of many similar systems using a variety of laser sources, all of which, however, must rely on external stabilization elements to maintain a constant $\mathrm{THz}$ frequency. Clearly, frequency stability and beam alignment can be more easily achieved using a single two-color laser. To this end, Tani et al. ${ }^{2}$ demonstrated THz photomixing of a multimode laser diode where the generated frequency is determined by the longitudinal mode spacing. Another scheme which achieved tuneable $\mathrm{THz}$ was developed by $\mathrm{Gu}$ et al. ${ }^{3}$ using a dual wavelength laser diode. The diode had an external resonator consisting of a diffraction grating and a V-shaped double-stripe end mirror which selected lasing modes. An all-optoelectronic CW THz imaging system was built by Siebert et al., ${ }^{4}$ employing a two-color Ti:sapphire laser ${ }^{5}$ with a dual cavity.

In this paper we describe a simple method of obtaining frequency-stable, tuneable two-mode laser operation by placing a Fabry-Perot etalon in the laser resonator. The difference frequency of the modes is then equal to the free spectral

${ }^{a)}$ Electronic mail: eenmrs@leeds.ac.uk range (FSR) of the etalon. The tuning can be either discrete, by changing fixed etalons, or continuous, by varying the etalon plate separation. The scheme eliminates the need for laser frequency stabilization and beam overlap alignment.

A variety of solid and air-gap low-finesse etalons were used to demonstrate the two-mode laser operation. Photomixing was performed in logarithmic spiral antennas fabricated on LT-GaAs, and the power and frequency of the emitted $\mathrm{CW} \mathrm{THz}$ radiation were measured. Photocurrent modulation in photomixers and their current-voltage $(I-V)$ curves were also observed.

\section{EXPERIMENTAL SETUP}

\section{A. The two-mode laser}

A Ti-sapphire laser was chosen to carry out the experiment, owing to several advantageous characteristics. A relatively compact air-cooled laser can produce power in excess of $0.5 \mathrm{~W}$. The operating wavelength is around $800 \mathrm{~nm}$, which is suitable for photomixing using GaAs devices. With a birefringent wavelength selector present in the cavity, the multimode emission bandwidth (comprising over $10^{4}$ longitudinal modes) is in excess of $10 \mathrm{~nm}$, allowing a frequency difference of up to $5 \mathrm{THz}$ to be generated. However, the single longitudinal mode linewidth, which determines the linewidth of the generated $\mathrm{THz}$ radiation, is of the order of only $50 \mathrm{kHz}$. This compares favorably with the linewidth of single-mode semiconductor diode lasers which is of the order of $10 \mathrm{MHz}$, indicating that the $\mathrm{THz}$ linewidth obtained in 


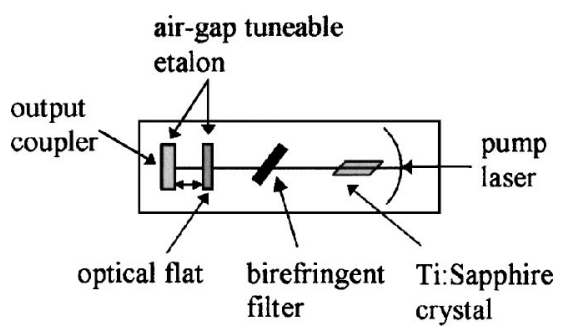

FIG. 1. Schematic diagram of the Ti:sapphire laser system.

two-laser frequency mixing experiments is broader than that achievable using a two-color Ti-sapphire laser.

A CW Ti:sapphire laser (Laser Quantum) was designed and custom built to support a tuneable two-mode operation. The laser produced $700 \mathrm{~mW}$ of infrared power, with a power stability of better than $1 \%$. The laser was tuneable from 750 to $850 \mathrm{~nm}$, using a birefringent filter, and had a longitudinal mode separation of $0.3 \mathrm{GHz}$. The two-mode operation was obtained by inserting a Fabry-Perot etalon into the laser cavity, as shown in Fig. 1, which forced the laser to oscillate on two (or more) modes separated by the free spectral range (FSR) of the etalon. The etalons used in the experiments were chosen so that the difference frequency of the modes was in the terahertz range.

Two types of Fabry-Perot etalons were employed: fixed and variable. Uncoated glass optical flats were used as fixed solid etalons with the FSRs of 0.16 and $0.56 \mathrm{THz}$. To obtain a $\mathrm{THz}$ frequency tuning, a variable air-gap etalon was also constructed by placing an optical flat mounted on a translation stage in front of the output coupler. This created an asymmetric etalon between the glass surface on one side and the mirror surface on the other. The outer surface of the glass flat was antireflection (AR)-coated to prevent double reflections.

All etalons, fixed and variable, had low finesse: 0.7 for the fixed and 3.8 for the variable. However, this was found to be sufficient for selecting laser modes separated by the FSR of the etalon and maintaining a stable two-mode operation. The laser modes were monitored by an optical spectrum analyzer (Ocean Optics) with a resolution of $0.1 \mathrm{~nm}$.

\section{B. Photocurrent modulation}

An autocorrelation-type measurement was used to observe photocurrent modulation due to two-mode mixing in a LT-GaAs chip. The beam from the two-mode laser was directed through a Michelson interferometer before being focused onto a LT-GaAs biased photomixer. The induced photocurrent in the photomixer was then measured as a function of the interferometer path difference. The observed modulation of the photocurrent therefore reflected the spatial variation of the optical intensity caused by the interference of the two laser modes. The large noise observable in the photocurrent signal was caused by mechanical instabilities in the pointing of the laser beam, which required it to be focused to an area of $<10 \mu \mathrm{m}^{2}$ on the photomixer chip.

\section{CW THz generation}

Continuous-wave $\mathrm{THz}$ radiation was generated by mixing the two-laser modes in logarithmic spiral antennas fabricated on LT-GaAs. The photomixer devices were all designed with an active area of $8 \times 8 \mu \mathrm{m}^{2}$ consisting of eight $0.2-\mu \mathrm{m}$ interdigitated fingers separated by $1-\mu \mathrm{m}$ gaps. The active region was formed in the center of a three-turn selfcomplimentary logarithmic spiral antenna, fabricated on 1$\mu$ m-thick LT-GaAs substrate with Ti:Au metallization. The driving-point impedance for this type of frequencyindependent antenna is $R_{\text {ant }}=72 \Omega$, calculated using the formula $R_{\text {ant }}=60 \pi /\left(\varepsilon_{\text {eff }}\right)^{1 / 2}$, where $\varepsilon_{\text {eff }}$ is the effective dielectric constant ( $\varepsilon_{\text {eff }} \approx 7$ for GaAs) (Ref. 6). The carrier lifetime of the LT-GaAs was $550 \mathrm{fs}$. All the photomixers were developed and fabricated at the Institute of Thin Films and Interfaces (ISG-1), Research Center Jülich, Jülich, Germany. ${ }^{7}$

The laser beam was attenuated to $<100 \mathrm{~mW}$ to avoid damaging the photomixers and was launched into a $800-\mathrm{nm}$ monomode optical fiber (Thorlabs, FSSN 4224). The diameter of the free-space Ti-sapphire beam was $1.5 \mathrm{~mm}$, and the coupling efficiency into the fiber was $<30 \%$. Owing to mechanical instabilities in the beam coupling into the fiber, the output power at the fiber exit fluctuated, giving rise to the noise in the $\mathrm{THz}$ signal. The fiber was passed through a polarization controller (Thorlabs, 973/579-7227) so that the polarization of the laser beam could be oriented precisely perpendicular to the photomixer electrodes. This is necessary in order to avoid undesirable reflections in the active region due to diffraction grating effects arising from the interdigitated finger structure. The fiber end was positioned close to the active region of the photomixer using a precision $x-y-z$ piezoelectric stage and a microscope objective. A dc bias voltage was then applied to the outer arms of the mixer structure through a low-pass filter circuit designed to eliminate voltage spikes. Since THz beams generated by photomixers have a large angular divergence, it was necessary to collimate the emitted beam. This was done by gluing a high resistivity silicon hyperhemispherical lens to the output surface of the photomixer chip.

The emitted $\mathrm{THz}$ power was determined by a calibrated Golay detector (QMC Instruments) equipped with a horn antenna to improve the collection efficiency of the $\mathrm{THz}$ radiation. The THz frequency was then measured using a Fouriertransform interferometer described elsewhere. ${ }^{8}$

\section{RESULTS AND DISCUSSION}

\section{A. Laser modes produced by intracavity Fabry-Perot etalons}

Figure 2 shows two examples of the laser spectra obtained with an intracavity Fabry-Perot etalon. Two settings of the variable air-gap etalon were used to produce a stable two-mode operation. The intensity ratio of the two modes varied from 1 to 0.6 , depending on the alignment of the laser and the etalon. 


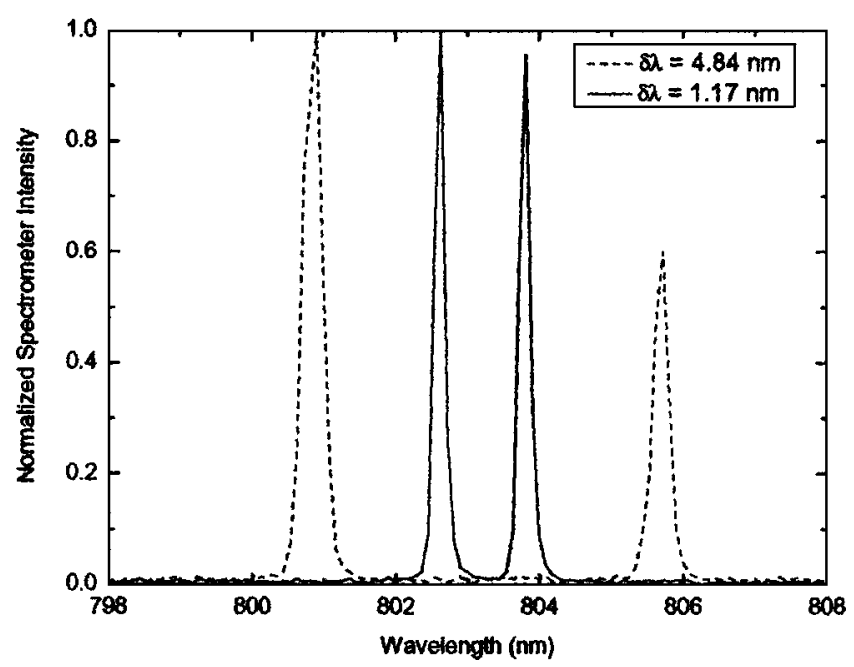

FIG. 2. Two-mode laser spectra obtained with a variable Fabry-Perot etalon.

\section{B. Photocurrent modulation}

The photocurrent modulation was observed using two solid glass etalons of 0.19 - and 0.6-mm thickness, giving the FSR of $0.56 \mathrm{THz}$ and $0.17 \mathrm{THz}$, respectively. The interferometer trace for the $0.19-\mathrm{mm}$ etalon is illustrated in Fig. 3(a). The fast oscillations in the trace are due to interference fringes arising from the laser wavelength of $800 \mathrm{~nm}$. The
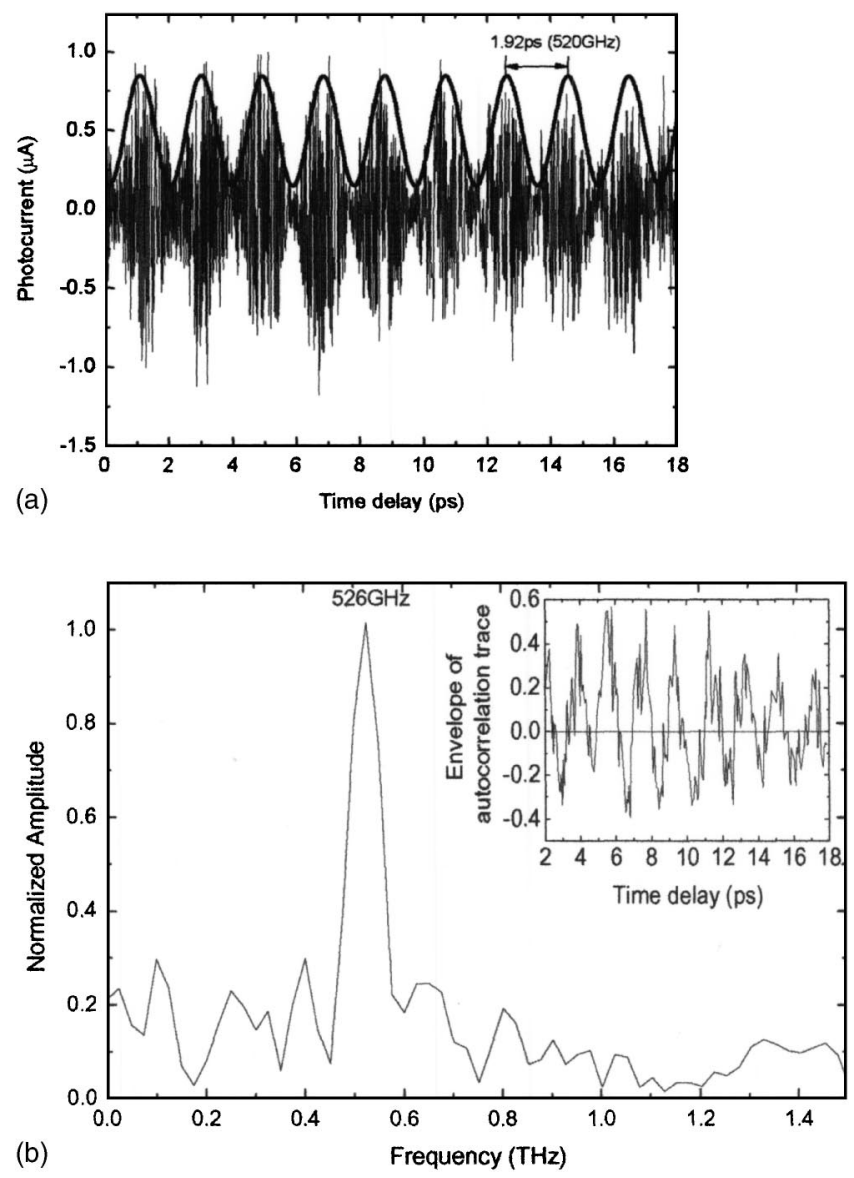

FIG. 3. Photocurrent modulation observed with a $0.19-\mathrm{mm}$ solid glass etalon: (a) an autocorrelation trace, (b) the FFT spectrum and the envelope of the autocorrelation trace (inset).

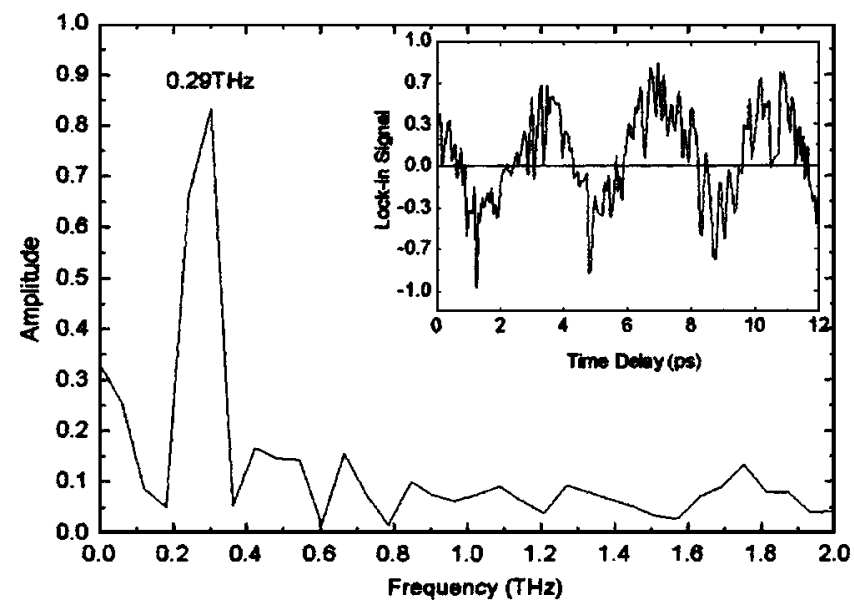

FIG. 4. A THz spectrum obtained with the Fourier-transform interferometer; inset: the interferometer trace.

$\mathrm{THz}$ modulation frequency was obtained from the data by defining the envelope of the interferometer trace and performing a fast Fourier transform (FFT), as shown in Fig. 3(b). The photocurrent modulation frequency is seen to correspond to the frequency difference of the laser modes, as given by the FSR of the etalon.

\section{THz frequency measurements}

The emitted $\mathrm{THz}$ frequency was measured using the $\mathrm{THz}$ Fourier-transform interferometer which is described elsewhere. ${ }^{8}$ An example of the interferometer trace and the calculated spectrum are shown in Fig. 4. The laser modes in this measurement were separated by $0.69 \mathrm{~nm}$, corresponding to a difference frequency of $0.29 \mathrm{THz}$. The interferometer trace therefore confirms that the observed $\mathrm{THz}$ radiation is generated by photomixing the two laser modes.

\section{Photomixer performance: The current-voltage and power-voltage curves}

The $I-V$ and $P_{\mathrm{THz}}$ curves of several photomixers were measured, and the results were analyzed using the relationships for the CW photomixers ${ }^{1,6}$

$$
\begin{aligned}
& I=\left(\frac{e}{h c} \lambda_{\mathrm{IR}} \mu_{e} \tau_{e} \eta \frac{N}{L}\right) P_{\mathrm{IR}} V_{\mathrm{bias}}, \\
& P_{\mathrm{THz}}=\frac{1}{2} Z_{\mathrm{ant}} \frac{I^{2}}{1+\omega_{\mathrm{THz}}^{2} \tau_{e}^{2}} \propto P_{\mathrm{IR}}^{2} V_{\mathrm{bias}}^{2} \frac{\tau_{e}^{2} \mu_{e}^{2}}{1+\omega_{\mathrm{THz}}^{2} \tau_{e}^{2}},
\end{aligned}
$$

where $I$ is the photocurrent, $V_{\text {bias }}$ is the bias voltage, $P_{\mathrm{IR}}$ and $\lambda_{\text {IR }}$ are the incident optical power and wavelength, respectively, and $P_{\mathrm{THz}}$ and $\omega_{\mathrm{THz}}$ are the emitted $\mathrm{THz}$ power and frequency, respectively. Carrier dynamics are expressed in terms of the mobility $\mu_{c}$ and lifetime $\tau_{e}$, and the emitter design via its impedance $Z_{\text {ant }}$, the number of interdigitated fingers $N$, the length of the active region $L$, and the quantum efficiency $\eta$.

Figure 5 shows the measured photocurrent as a function of applied bias for two similar antennas, obtained using different settings of the laser wavelength. The maximum photocurrent was limited to $1 \mathrm{~mA}$ in order to avoid thermal and 


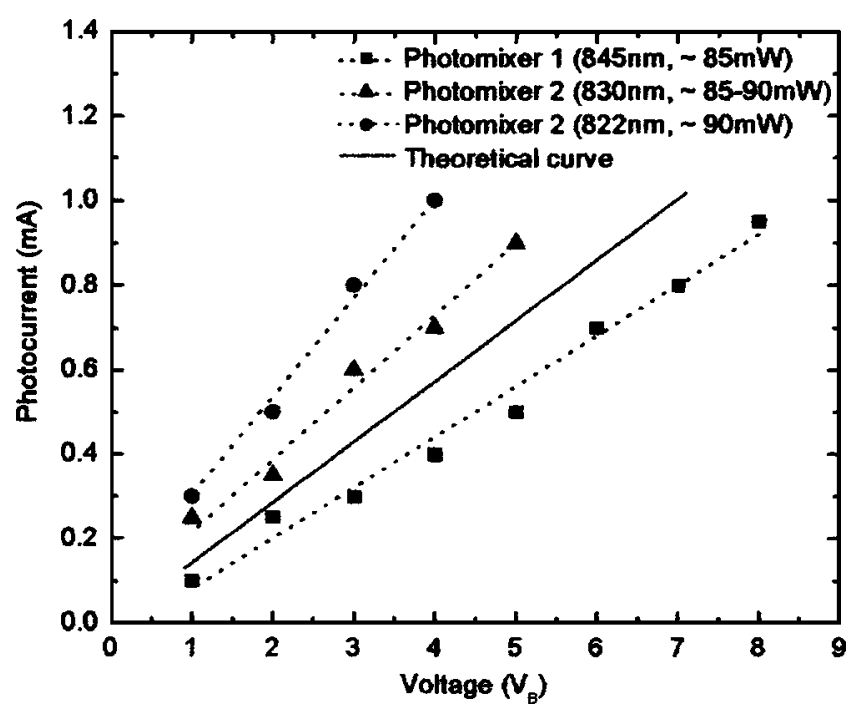

FIG. 5. Photomixer current-voltage relationship for different settings of the laser wavelength.

electrical breakdown of the photomixer, which would result in permanent damage to the device. ${ }^{6}$ As expected, a linear relationship between the photocurrent and the applied voltage was observed in all the measurements.

A theoretical $I-V$ curve, calculated using Eq. (1) and the known photomixer parameters is also plotted in Fig. 5 for comparison (solid line). The parameters used were: $P_{\mathrm{IR}}$ $=85 \mathrm{~mW}, \quad \lambda_{\mathrm{IR}}=845 \mathrm{~nm}, \quad \tau_{e}=550 \mathrm{fs}, \quad$ and $\mu_{e}$ $=167 \mathrm{~cm}^{2} \mathrm{~V}^{-1} \mathrm{~s}^{-1}, 5, \eta=0.325, N=8$, and $L=8.6 \mu \mathrm{m}$. The calculated $I-V$ relationship is seen to be similar to the measured values. Since there are significant uncertainties in several of the parameters used in the calculation (e.g., in $\eta$ ), a precise agreement between the calculation and the experiment is not expected.

The $\mathrm{THz}$ power emitted was also measured as a function of the bias voltage. The maximum power observed at 1$\mathrm{mA}$ photocurrent was $0.9 \mu \mathrm{W}$ at $0.3 \mathrm{THz}$ and $0.7 \mu \mathrm{W}$ at $0.5 \mathrm{THz}$. Figure 6 plots the THz power at $0.3 \mathrm{THz}$ against the bias voltage for two different photomixers. A square-law relationship is observed in both cases, in agreement with Eq. (2). A theoretical curve calculated using Eq. (2) and the parameters above is also included for comparison, and is seen to be similar to the observed behavior.

\section{CONCLUSIONS}

We have demonstrated a simple method of achieving a two-mode operation in a Ti:sapphire laser for the purposes of generating a $\mathrm{CW} \mathrm{THz}$ radiation. Two stable modes separated by a difference frequency in the $\mathrm{THz}$ range were obtained by inserting a suitable Fabry-Perot etalon into the laser resona-

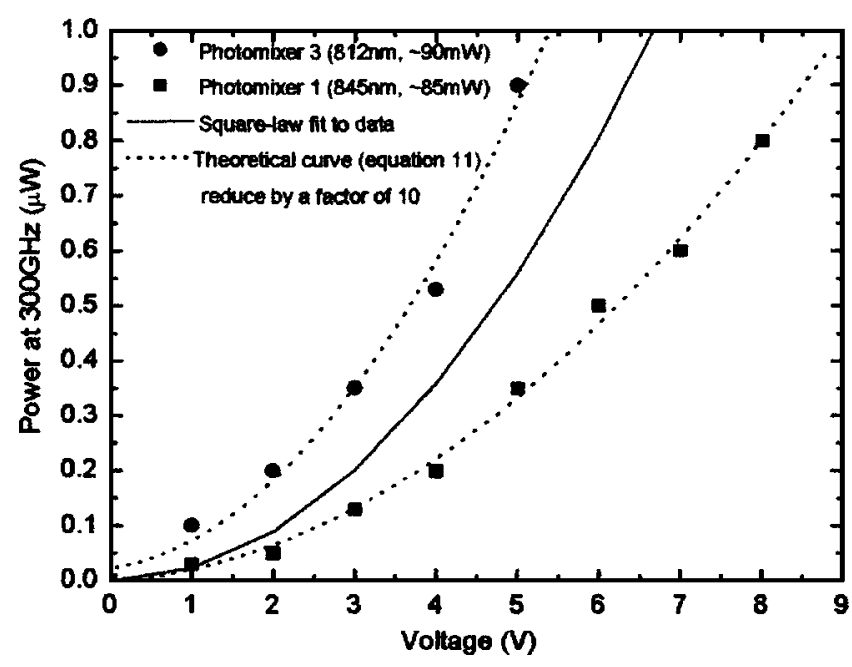

FIG. 6. Power emitted at $0.3 \mathrm{THz}$ as a function of the bias voltage.

tor. Compared with two-laser systems, the two-mode laser has advantages of better $\mathrm{THz}$ frequency stability with no need for beam overlap alignment.

The CW $\mathrm{THz}$ radiation was produced by photomixing the two laser modes in a logarithmic spiral antenna fabricated on LT-GaAs. A difference frequency generation was confirmed and photomixer performance was characterized by measurements of $\mathrm{THz}$ power and frequency, photocurrent modulation, and $I-V$ curves. The photomixer behavior was found to conform to the model. The emitted $\mathrm{THz}$ power of up to $0.9 \mu \mathrm{W}$ at $0.3 \mathrm{THz}$ was observed.

\section{ACKNOWLEDGMENTS}

This project was supported by UK Research Council's Basic Technology Research Grant No. GR/R88106/01 and EPSRC studentship.

${ }^{1}$ E. R. Brown, F. W. Smith, and K. A. McIntosh, J. Appl. Phys. 73, 1480 (1993).

${ }^{2}$ M. Tani, S. Matsuura, K. Sakai, and M. Hangyo, IEEE Microw. Guid. Wave Lett. 7, 282 (1997).

${ }^{3}$ P. Gu, F. Chang, M. Tani, K. Sakai, and C.-L. Pan, Jpn. J. Appl. Phys., Part 2 38, 1246 (1999).

${ }^{4}$ K. J. Siebert, H. Quast, R. Leonhardt, T. Löffler, M. Thomson, T. Bauer, and H. G. Roskos, Appl. Phys. Lett. 80, 3003 (2002).

${ }^{5}$ F. Siebe, K. J. Siebert, R. Leonhardt, and H. G. Roskos, IEEE J. Quantum Electron. 35, 1731 (1999).

${ }^{6}$ E. R. Brown, Electronic Devices and Advanced Systems Technology, in Terahertz Sensing Technology Vol. 1, edited by D. L. Woolard, W. R. Loerop, and M. S. Shur (World Scientific, Singapore, 2003).

${ }^{7}$ I. C. Mayorga, M. Mikulics, A. Schmitz, P. V. der Wal, R. Guesten, M. Marso, P. Kordos, and H. Luth, Proceedings of SPIE in Millimeter and Submillimeter Detectors for Astronomy II, edited by J. Zmuidzinas, W. S. Holland, and S. Withington, Vol. 5498, p. 537-547 (2004).

${ }^{8}$ H. Eisele, M. Naftaly, J. R. Fletcher, D. P. Steenson, and M. R. Stone, Proceedings of the 15th International Symposium on Space Terahertz Technology, Northampton, MA, 27-29 May 2004 (unpublished). 